\title{
Tuberculin conversion and tuberculosis disease in infants and young children from the Drakenstein Child Health Study: A call to action
}

Although tuberculosis (TB) is widely acknowledged as a major driver of global morbidity and mortality in adults, the disease's impact on children has been underappreciated. Global estimates of mortality among children aged $<5$ years, derived largely from vital registration and verbal autopsy records, have excluded paediatric TB as a contributing cause. ${ }^{[1,2]}$ Recent modelling studies, however, have estimated that TB is a leading cause of childhood morbidity and mortality, causing $\sim 1$ million new cases and 250000 deaths per year. ${ }^{[2-5]}$ Approximately $80 \%$ of these deaths occur before 5 years of age and many are never diagnosed. ${ }^{[3]}$

Our lack of knowledge regarding paediatric TB is driven by several factors that make diagnosis of children with TB challenging in lowand middle-income countries (LMICs), where most paediatric TB patients reside. Nonspecific symptoms and poor sensitivity of tests or lack of training or capacity for microbiological testing, such as sputum culture and GeneXpert MTB/RIF, hamper diagnosis. ${ }^{[6,7]}$ Most diagnostic procedures, such as chest radiographs and tuberculin skin testing, have reduced value in HIV-infected paediatric populations. ${ }^{[7]}$ In addition, surveillance systems in resource-constrained settings are not equipped to detect paediatric $\mathrm{TB}$ owing to lack of laboratory infrastructure or tuberculin shortages. Consequently, much paediatric TB is undiagnosed in LMIC settings, and programmatic estimates (from case notification, vital registration or verbal autopsy) of TB disease in young children are likely to be substantial underestimates of the true community burden.

Improving our understanding of the epidemiology of TB in infancy and young childhood in high-burden settings is critical to inform national and global guidelines and devise interventions that target paediatric populations at high risk for TB and subsequent mortality. To fill this knowledge gap, birth cohorts are urgently needed.

The Drakenstein Child Health Study is a South African (SA) birth cohort study of 1137 mother-child pairs investigating the early-life determinants of child health and epidemiology of several important determinants of health, including childhood pneumonia, wheezing, lung function and TB. ${ }^{[8]}$ The study is being conducted in a periurban area of Paarl, outside Cape Town. Pregnant women at 22 - 28 weeks' gestation were enrolled at two healthcare clinics and followed until childbirth; their offspring have then been followed through childhood. Tuberculin skin tests were administered at 6,12 , 24, 36, 48 and 60 months of age, and also when children presented with a lower respiratory tract infection or pneumonia. Continuous $\mathrm{TB}$ and pneumonia surveillance is conducted by trained nurses and clinicians. ${ }^{[9]}$ There is a strong local primary healthcare system in the area where all participants access healthcare. Almost a quarter of pregnant women in the cohort were living with HIV; however, owing to widespread antiretroviral therapy preventing vertical transmission of HIV, only two children $(<1 \%)$ tested HIV-positive. All children included in the cohort received BCG vaccination at birth.

Our objective with this study was to evaluate the incidence and burden of TB infection and disease in the first 5 years of life in this birth cohort. We also wanted to identify prenatal and early childhood risk factors for TB-related outcomes. ${ }^{[10]}$

We defined tuberculin conversion as an induration reaction of $\geq 10 \mathrm{~mm}$, as recommended by national and global guidelines. ${ }^{[1,12]}$
This induration cut-off is considered conservative and is used to avoid false-positive responses resulting from non-tuberculous mycobacterial infection or BCG vaccination. Boosting of tuberculin results is possible when recurrent skin tests are administered, as was done in our study. To prevent misclassification of boosted reactions, we did not administer additional skin tests to children with a positive test or a reactive skin test between 1 and $9 \mathrm{~mm}$. These children were subsequently censored in further analyses of tuberculin conversion. Children who tested positive were subsequently referred to local TB clinics for preventive therapy; however, study investigators could not enforce prescription and completion. We used three distinct definitions of TB disease: (i) all diagnosed TB disease; (ii) radiographically and microbiologically confirmed TB; and (iii) microbiologically confirmed TB only.

We found high rates of tuberculin conversion (11.8 conversions per 100 child-years) and all diagnosed TB disease (2.9 paediatric cases per 100 child-years). These rates equate to an $\sim 11.8 \%$ annual risk of infection and 2900 paediatric cases per 100000 children, among the highest reported estimates of paediatric TB globally. When we restricted our definition of $\mathrm{TB}$ disease to only microbiologically confirmed TB, which has low sensitivity in young children, we found a high rate of 0.7 paediatric cases per 100 child-years (700 paediatric cases per 100000 children). We also found novel risk factors for TB disease such as lower respiratory tract infection (adjusted hazard ratio (AHR) 2.3, 95\% confidence interval (CI) 1.4 - 3.6; $p<0.0001$ ) or maternal smoking during pregnancy (AHR 1.7, 95\% CI 1.0 - 2.8; $p=0.04$ ). Children who received isoniazid (INH) preventive therapy were highly protected against developing disease (AHR 0.2, 95\% CI $0.1-0.6 ; p<0.0001$ ), but use of INH prophylaxis among tuberculin converters was disappointingly low, with only $22 \%$ completion.

Results from the Drakenstein cohort elucidate several characteristics regarding the epidemiology of paediatric TB in SA and other areas of sub-Saharan Africa. First, the high rates of TB infection and disease we observed indicate that delivery of multipronged interventions that prevent disease acquisition and increase TB case detection in young children are urgently needed. Although increased awareness has been given to paediatric TB by national and global guidelines in the past decade ${ }^{[11-13]}$ strengthening of childhood TB services to under-resourced communities has not followed suit. ${ }^{[14]}$ Second, young children are continuously exposed to $\mathrm{TB}$ and probably reinfected several times throughout their first years of life in these settings, ${ }^{[10,15]}$ indicating that interventions must be lasting, sustainable and costeffective. Third, our finding that a relationship exists between lower respiratory tract infection and $\mathrm{TB}$ disease confirms prior studies showing high rates of confirmed pulmonary $\mathrm{TB}$ in children with severe pneumonia. ${ }^{[16-18]}$ Our study confirms that the association is present, and that these two diseases are strongly interconnected in young children at the community level.

The alarming childhood TB burden we see in the Drakenstein cohort, despite a strong primary healthcare system including wide healthcare access, comprehensive antiretroviral therapy and universal BCG vaccination, suggests that paediatric TB control must become a major public health priority in SA and other sub-Saharan African countries. Children rarely present to $\mathrm{TB}$ clinics, so integration 
of paediatric TB services with control of other diseases, such as maternal and child health facilities or sick visits for lower respiratory tract infections, is an opportunity to improve detection of paediatric $\mathrm{TB}^{\left[{ }^{[3]}\right.}$ This approach will need to be partnered with a push to improve policy-practice gaps, laboratory infrastructure, use of and adherence to INH prophylaxis, and clinical training to increase paediatric case detection and reduce paediatric mortality in Africa. Such a comprehensive package is essential to reduce the childhood TB burden in Africa and other LMICs. There is great urgency to implement interventions that combine detection, treatment and prevention of TB while maintaining resource efficiency.

Acknowledgements. We thank the study staff and the clinical and administrative staff of the Western Cape Government Health Department at Paarl Hospital and at the clinics for support of the study. We also thank members of the study International Advisory Board for their advice, our collaborators, and the families and children who participated in the study. Funding. This study was funded by the Bill \& Melinda Gates Foundation (grant number OPP 1017641), the South African Medical Research Council, and the National Research Foundation of SA.

\section{Leonardo Martinez}

Division of Infectious Diseases and Geographic Medicine, School of Medicine, Stanford University, Calif., USA

\author{
Heather J Zar \\ Department of Paediatrics and Child Health, \\ Red Cross War Memorial Children's Hospital \\ and MRC Unit on Child and Adolescent \\ Health, Faculty of Health Sciences, \\ University of Cape Town, South Africa. \\ heather.zar@uct.ac.za
}
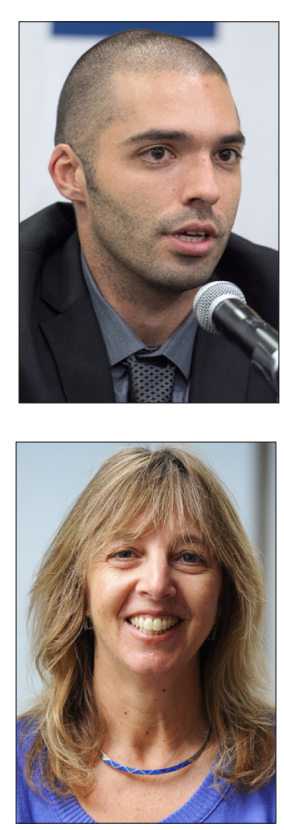

1. Liu L, Johnson HL, Cousens S, et al. Global, regional, and national causes of child mortality: An updated systematic analysis for 2010 with time trends since 2000. Lancet 2012;379(9832):2151-2161. updated systematic analysis for 2010 with time trend

2. Liu L, Oza S, Hogan D, et al. Global, regional, and national causes of under-5 mortality in $2000-15$ : An updated systematic analysis with implications for the Sustainable Development Goals. Lancet 2017;388(10063):3027-3035. https://doi.org/10.1016/\$0140-6736(16)31593-8

3. Dodd P, Yuen C, Sismanidis C, Seddon J, Jenkins H. The global burden of tuberculosis mortality in children: A mathematical modelling study. Lancet Glob Health 2017;5(9):e898-e906. https://doi. org/10.1016/S2214-109X(17)30289-9

4. Dodd PJ, Gardiner E, Coghlan R, Seddon JA. Burden of childhood tuberculosis in 22 high-burden countries: A mathematical modelling study. Lancet Glob Health 2014;2(8):e453-e459. https://doi. org/10.1016/S2214-109X(14)70245-1

5. Jenkins HE, Tolman AW, Yuen CM, et al. Incidence of multidrug-resistant tuberculosis disease in children: Systematic review and global estimates. Lancet 2014;383(9928):1572-1579. https://doi. children: Systematic review and glob
org/10.1016/S0140-6736(14)60195-1

6. Schumacher SG, van Smeden M, Dendukuri N, et al. Diagnostic test accuracy in childhood pulmonary tuberculosis: A Bayesian latent class analysis. Am J Epidemiol 2016;184(9):690-700. https://doi.org/10.1093/aje/kww094

7. Nicol MP, Zar HJ. New specimens and laboratory diagnostics for childhood pulmonary TB: Progress and prospects. Paediatr Respir Rev 2011;12(1):16-21. https://doi.org/10.1016/j.prrv.2010.09.008

8. Zar H, Barnett W, Myer L, Stein D, Nicol M. Investigating the early-life determinants of illness in Africa: The Drakenstein Child Health Study. Thorax 2014;79(6):592-594. https://doi.org/10.1136/ thoraxinl-2014-206242

9. Le Roux DM, Myer L, Nicol MP, Zar HJ. Incidence and severity of childhood pneumonia in the first year of life in a South African birth cohort: The Drakenstein Child Health Study. Lancet Glob Health 2015;3(2):e95-e103. https://doi.org/10.1016/S2214-109X(14)70360-2

10. Martinez L, le Roux DM, Barnett W, Stadler A, Nicol MP, Zar HJ. Tuberculin skin test conversion and primary progressive tuberculosis disease in the first 5 years of life: A birth cohort study from Cape Town, South Africa. Lancet Child Adolesc Health 2018;2(1):46-55. https://doi.org/10.1016/ S2352-4642(17)30149-9

11. National Department of Health, South Africa. Guidelines for the Management of Tuberculosis in Children. Pretoria: NDoH, 2013.

12. World Health Organization. Guidance for National Tuberculosis Programmes on the Management of Tuberculosis in Children. Geneva: WHO, 2014.

13. World Health Organization. Guidance for National Tuberculosis Programmes on the Management of Tuberculosis in Children. Geneva: WHO, 2006.

of Tuberculosis in Children. Geneva: WHO, 2006.
4. Hill PC, Rutherford ME, Audas R, van Crevel R, Graham SM. Closing the policy-practice gap in the management of child contacts of tuberculosis cases in developing countries. PLoS Med 2011;8(10):e1001105. https://doi.org/10.1371/journal.pmed.1001105

15. Martinez L, Shen Y, Handel A, et al. Effectiveness of WHO's pragmatic screening algorithm for child contacts of tuberculosis cases in resource-constrained settings: A prospective cohort study in Uganda. Lancet Respir Med 2017 (epub 19 December 2018). https://doi.org/10.1016/S22132600(17)30497-6

16. Moore DP, Klugman KP, Madhi SA. Role of Streptococcus pneumoniae in hospitalization for acute community-acquired pneumonia associated with culture-confirmed Mycobacterium tuberculosis in children: A pneumococcal conjugate vaccine probe study. Pediatr Infect Dis J 2010;29(12):10991104. https://doi.org/10.1097/INF.0b013e3181 eaefff

17. Zar H, Hanslo D, Tannenbaum E, et al. Aetiology and outcome of pneumonia in human immunodeficiency virus-infected children hospitalized in South Africa. Acta Paediatr 2001;90(2):119-125. https://doi.org/10.1111/j.1651-2227.2001.tb00270.x

18. Oliwa JN, Karumbi JM, Marais BJ, Madhi SA, Graham SM. Tuberculosis as a cause or comorbidity of childhood pneumonia in tuberculosis-endemic areas: A systematic review. Lancet Respir Med 2015;3(3):235-243. https://doi.org/10.1016/\$2213-2600(15)00028-4 